This document is confidential and is proprietary to the American Chemical Society and its authors. Do not copy or disclose without written permission. If you have received this item in error, notify the sender and delete all copies.

\title{
Inhibition and regulation of the ergothioneine biosynthetic methyltransferase EgtD
}

\begin{tabular}{|r|l|}
\hline Journal: & ACS Chemical Biology \\
\hline Manuscript ID & cb-2018-00127c.R2 \\
\hline Manuscript Type: & Article \\
\hline Date Submitted by the Author: & 06-Apr-2018 \\
\hline Complete List of Authors: & $\begin{array}{l}\text { Misson, Laetitia; University of Basel, Department for Chemistry } \\
\text { Burn, Reto; University of Basel, Department for Chemistry } \\
\text { Vit, Allegra; Helmholtz Zentrum für Infektionsforschung, Struktur und } \\
\text { Funktion der Proteine } \\
\text { Hildesheim, Juila; University of Basel, Department for Chemistry } \\
\text { Beliaeva, Mariia; University of Basel, Department for Chemistry } \\
\text { Blankenfeldt, Wulf; Helmholtz Zentrum für Infektionsforschung, Struktur } \\
\text { und Funktion der Proteine } \\
\text { Seebeck, Florian; University of Basel, Department for Chemistry }\end{array}$ \\
\hline
\end{tabular}

SCHOLARONE

Manuscripts 


\title{
Inhibition and regulation of the ergothioneine biosynthetic methyltransferase EgtD
}

Laetitia Misson,1,\& Reto Burn, ${ }^{1, \&}$ Allegra Vit, ${ }^{2, \&}$ Julia Hildesheim, ${ }^{1}$ Mariia A. Beliaeva, ${ }^{1}$ Wulf Blankenfeldt ${ }^{2,3}$ and Florian P. Seebeck ${ }^{1 *}$

${ }^{1}$ Department for Chemistry, University of Basel, BPR 1096, Mattenstrasse 24a, Basel, Switzerland ${ }^{2}$ Structure and Function of Proteins, Helmholtz Centre for Infection Research, Inhoffenstr. 7, 38124, Braunschweig, Germany

${ }^{3}$ Institute for Biochemistry, Biotechnology and Bioinformatics, Technische Universität Braunschweig, 38106 Braunschweig, Germany

*To whom correspondence should be addressed: florian.seebeck@unibas.ch \&These authors contributed equally to this work

\begin{abstract}
Ergothioneine is an emerging factor in cellular redox homeostasis in bacteria, fungi, plants and animals. Reports that ergothioneine biosynthesis may be important for the pathogenicity of bacteria and fungi raise the question as to how this pathway is regulated and whether the corresponding enzymes may be therapeutic targets. The first step in ergothioneine biosynthesis is catalyzed by the methyltransferase EgtD that converts histidine into N- $\alpha$-trimethylhistidine. This report examines the kinetic, thermodynamic and structural basis for substrate, product and inhibitor binding by EgtD from Mycobacterium smegmatis. This study reveals an unprecedented substrate binding mechanism and a fine-tuned affinity landscape as determinants for product specificity and product inhibition. Both properties are evolved features that optimize the function of EgtD in the context of cellular ergothioneine production. Based on these findings we developed a series of simple histidine derivatives that inhibit methyltransferase activity at low micromolar concentrations. Crystal structures of inhibited complexes validate this structure- and mechanism-based design strategy.
\end{abstract}

Keywords. Methyltransferase, inhibitor design, mechanism, oxidative stress Introduction

Ergothioneine (EGT, Figure 1), the betaine of 2-mercaptohistidine, is a ubiquitous metabolite. Many bacteria $^{1-5}$ and most fungi biosynthesize EGT.1, 6 Plants and animals absorb EGT from their environment through a dedicated EGT transporter protein. ${ }^{7,8}$ Active procurement of EGT by such a 
diverse array of organisms indicates the EGT may play a fundamental role in cellular life. This hypothesis is more than half a century old but is now being tested and debated with increasing

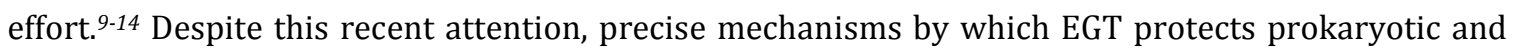
eukaryotic cells are still elusive.11,13,14 The unusual redox activity and metal binding properties of the mercaptoimidazole side chain ${ }^{15-18}$ could enable EGT to participate in a broad range of processes ${ }^{19}$ including protection against reactive oxygen species, ${ }^{20}$ reduction of oxidized heme-proteins, ${ }^{21}$ or passivating redox-active transition metals. ${ }^{22,23}$

Cellular dependence on EGT has been demonstrated for several microbial organisms. ${ }^{24}$ Deletion of EGT biosynthetic genes in Mycobacterium smegmatis, Mycobacterium tuberculosis, Streptomyces coelicolor, Neurospora crassa and Aspergillus fumigatus produced strains with reduced resistance against oxidative stress. ${ }^{25-31}$ In $M$. tuberculosis these deletions increased susceptibility to antimycobacterial drugs, and decreased viability in macrophages and in mice. ${ }^{30}$ These recent findings raise the possibility that EGT biosynthesis - a process that does not occur in human cells - may be a target for novel antiinfective therapeutics. The genetic studies also agree that mutating the gene for the S-adenosylmethionine (SAM) dependent methyltransferase EgtD induces complete EGT deficiency in bacteria and fungi. This dependence validates EgtD as a potential target for EGT biosynthesis inhibitors.

EgtD initiates EGT biosynthesis by methylating histidine (HIS) to produce N- $\alpha$-trimethylhistidine (TMH) via the intermediates $\mathrm{N}-\alpha$-monomethyl- (MMH) and $\mathrm{N}$ - $\alpha$-dimethylhistidine (DMH). ${ }^{2,32,33} \mathrm{TMH}$ is consumed by the oxygen- and iron-dependent sulfoxide synthase EgtB. This enzyme attaches the sulfur atom of $\gamma$-glutamylcysteine $(\gamma \mathrm{GC})$ to carbon 2 on the imidazole ring of TMH. ${ }^{34,35}$ Subsequent steps catalyzed by the amidohydrolase EgtC and the $\beta$-lyase EgtE result in EGT (Figure 1).36,37 Fungal homologs of EgtB utilize cysteine instead of $\gamma \mathrm{GC}$ as sulfur donor, but the chemistry of this reaction is likely similar to that of mycobacterial enzymes. ${ }^{27,} 38-40$ Some cyanobacterial species recruited a homologous iron-dependent enzyme from a different pathway to act as an EgtB surrogate in EGT production. ${ }^{41}$ An even more surprising variation of this pathway occurs in anaerobic green sulfur bacteria. These organisms utilize a rhodanese-like enzyme (EanB) to attach sulfur to TMH in an oxygen-independent reaction..$^{5}$ All these pathway variations include an EgtD-type methyltransferase, making this enzyme the sole indispensable component of EGT production (Figure 1).

Figure 1. Four biosynthetic pathways for ergothioneine (EGT) production in mycobacteria, ${ }^{2}$ fungi, ${ }^{27,38-40}$ cyanobacteria, ${ }^{41}$ and anaerobic green sulfur bacteria. ${ }^{5}$

The growing recognition of EGT as a relevant factor in microbial metabolism and the key role of EgtD in EGT biosynthesis motivated us to examine the kinetic, thermodynamic and structural basis for 
Figure 2. Lineweaver-Burk plots of kinetic data used to examine the substrate binding mechanism of EgtD. Top: Primary and secondary plots with SAM as the variable substrate in presence of different concentrations of HIS.

Bottom: Primary and secondary plots with HIS as the variable substrate in presence of different concentrations of SAM.

An obligatory binding order is consistent with the structure of EgtD (Figure 3). The active site of this enzyme is located in a cleft between the SAM-binding Rossmann-fold domain and the HIS-binding domain. The first domain is conserved among class I methyltransferases, ${ }^{44,45}$ but the second domain is exclusive to Methyltransf_33 enzyme family members, such as the Trp-, Tyr- and dimethylallyltryptophan methyltransferases..$^{33,46}$ 
The crystal structure of EgtD in complex with DMH and SAH shows that the enzyme completely sequesters the methyl acceptor from bulk solvent (Figure 3). The only direct non-protein contact to DMH is provided by the sulfur atom of SAH. The only path for HIS in and out of this pocket leads through the SAM/SAH-binding site. Unless substrate binding is accompanied by large scale unfolding and refolding of the HIS-binding domain, the methyl-acceptor can reach its binding pocket only in the absence of SAM/SAH. Therefore substrate binding and product release must follow an ordered sequence.

This methyl-acceptor first binding order distinguishes EgtD from all characterized natural product methyltransferases. ${ }^{47}$ Methyltransferases which methylate small substrates ${ }^{47}$ usually follow a SAMfirst or a random binding mechanism. Some DNA-, RNA- or protein-methyltransferases may follow an apparent substrate-first binding mechanism. ${ }^{48}$ However, these enzymes often bind their macromolecular substrates through interactions outside the active side, which makes the comparison to enzymes with small substrates difficult.

Figure 3: Structure of EgtD in complex with S-adenosyl homocysteine (SAH, green) and N- $\alpha$-dimethylhistidine (DMH, orange)(PDB: 4PIO).5 The substrate-binding domain (blue) is formed by residues $1-60$ and $196-286$. The SAM-binding domain is conserved in most SAM-dependent methyltransferases.

Product inhibition by TMH. EgtD is characterized by significant HIS-competitive inhibition by the product TMH. This behavior is also unusual for a SAM-dependent methyltransferase. To examine this trait of EgtD we recorded methyl-transfer activities in the presence of several TMH concentrations with either HIS or SAM as the substrate with variable concentration, while keeping the second substrate concentration constant. Plotting this data in form of Lineweaver-Burk plots showed that TMH behaves as a competitive inhibitor with respect to both substrates (Figure 4). The $K_{\mathrm{i}}$ for HIScompetitive inhibition of EgtD by TMH was determined by measuring the apparent $K_{\mathrm{M}, \mathrm{HIS}}$ in the presence of $500 \mu \mathrm{M}$ SAM and three different concentrations of TMH. From this data $K_{\mathrm{i}}$ was calculated using the equation $K_{\mathrm{i}}=K_{\mathrm{M}}[\mathrm{TMH}] /\left(K_{\mathrm{M}, \text { app }}-K_{\mathrm{M}}\right)($ Table 1 , Figure S2).

Table 1. Inhibition constants $\left(K_{\mathrm{i}}\right)$ for EgtD inhibitors ${ }^{[a]}$

\begin{tabular}{c|c|c} 
EgtD ligands & $\begin{array}{c}K_{\mathrm{i}}(\mu \mathrm{M}) \\
\text { L-derivative }\end{array}$ & $\begin{array}{c}K_{\mathrm{i}}(\mu \mathrm{M}) \\
\text { racemic }\end{array}$ \\
\hline TMH & $39 \pm 6$ & - \\
1 & - & $8.5 \pm 2.1$ \\
2 & $21 \pm 3$ & $41 \pm 6$ \\
3 & - & $93 \pm 11$ \\
4 & - & $49 \pm 14$
\end{tabular}


The value of $K_{\mathrm{i}}$ for TMH corresponds well with the dissociation constant $\left(K_{\mathrm{d}}\right)$ of the EgtD:TMH complex as determined by isothermal titration calorimetry (Table 2, Figure S3). The affinity of EgtD for TMH increased by 240 -fold in the presence of $7 \mathrm{mM} \mathrm{SAH} \mathrm{(Figure} \mathrm{S3).} \mathrm{However,} \mathrm{because} \mathrm{the}$ reaction mixtures used for the kinetic measurements contained SAH nucleosidase and adenine deaminase, SAH cannot accumulate, ${ }^{43}$ and does not contribute to inhibition. Similarly, the SAH concentrations in living cells is also kept in the in the low micromolar range, suggesting that EgtD inhibition by SAH may not be significant in vivo. ${ }^{49}$ On the other hand, stress factors that lead to accumulation of SAH might indeed interfere with EGT production.

Figure 4. Lineweaver-Burk plots of the data used to examine EgtD inhibition by TMH and 8. Top: Primary plots with HIS or SAM as the variable substrate in presence of different concentrations of TMH Bottom: Primary plots with HIS or SAM as the variable substrate in presence of different concentrations of $\mathbf{8}$.

Comparison to related methyltransferases. Methyltransferases are commonly inhibited by the consumed methyl donor, S-adenosyl homocysteine (SAH), which acts as a SAM-competitive inhibitor. ${ }^{49}$ Inhibition by the methylated product is far less common among methyltransferases. 
Therefore we wondered whether product inhibition by TMH is a specifically evolved feature of EgtD or whether this behavior is a mere consequence of the unusual substrate binding order or of the permethylation reaction. To address this question, two close EgtD homologs were examined. The first enzyme is the tyrosine betaine synthase (Ybs) from Aspergillus nidulans. Although Ybs shares only 28 $\%$ sequence identity with EgtD, ${ }^{33}$ this fungal enzyme contains an almost identical set of active site residues. The only apparent differences between EgtD and Ybs map to the side chain binding pocket for the substrate. Hence, Ybs and EgtD should share all catalytic properties that are inescapable consequences of the protein architecture or the catalyzed reaction.

Using the same kinetic assay as described above we determined that Ybs catalyzes tyrosine methylation with similar efficiency as EgtD catalyzed methylation of HIS (Table 3, Figure S4). In contrast to EgtD, Ybs is not inhibited by its final product $\mathrm{N}$ - $\alpha$-trimethyltyrosine (TMY, $K_{\mathrm{i}}>1 \mathrm{mM}$, Figure S5). The efficiencies at which the two enzymes catalyze the conversion of DMH or DMY to TMH or TMY, respectively, were also determined. EgtD-catalyzed methyl transfer is three-fold more efficient when the methyl acceptor is DMH instead of HIS. ${ }^{33}$ In contrast, Ybs-catalyzed methyl transfer is four-fold less efficient when the methyl acceptor is DMY instead of Tyr. As a consequence, EgtD and Ybs give rise to different product distributions when SAM is the limiting substrate. EgtD produces predominantly TMH, while Ybs produces predominantly DMY (Table 3).

Similar observations were made with an engineered EgtD variant (EgtD $\left.D_{\mathrm{E} 282 \mathrm{~A}, \mathrm{M} 252 \mathrm{~V}}\right)$ that methylates tryptophan instead of HIS. ${ }^{33}$ The variant contains two mutations in the substrate-binding domain that accommodate an indole instead of an imidazole ring. The crystal structure of this enzyme in complex with tryptophan (Trp) revealed an otherwise unchanged active site geometry. ${ }^{33}$ EgtD E282A,M252V $_{2}$ catalyzed methylation of Trp with an efficiency only six-fold lower than that of the wild type enzyme with HIS as substrate (Table 3, Figure S6). However, methylation of $\mathrm{N}$ - $\alpha$-dimethyltryptophan (DMW) to $\mathrm{N}$ - $\alpha$-trimethyltryptophan (TMW) is 20 -fold less efficient than the corresponding transformation of DMH by EgtD. The reduced efficiency is due to a reduced $k_{\text {cat }}$, suggesting that suboptimal positioning of the non-native substrate in the mutated active site specifically affects methyl transfer to DMW.

Based on the comparison of these three methyltransferases we conclude that efficient trimethylation and product inhibition as observed by EgtD are not inescapable consequences of the active site architecture, the catalyzed reaction type, or the substrate binding mechanism. More likely, the two behaviors rely on structural optimization of the EgtD active site and must have emerged by positive selection to serve a function. As will be discussed below, cooperative trimethylation and product inhibition may play important roles in quality control and regulation of EGT biosynthesis in Mycobacteria. 
Table 3. Kinetic parameters of the aromatic amino acid betaine synthases a

\begin{tabular}{|c|c|c|c|c|c|}
\hline substrates & enzyme & $\begin{array}{l}k_{\text {cat }} \\
\left(\mathrm{s}^{-1}\right)\end{array}$ & $\begin{array}{c}K_{\mathrm{M}} \\
(\mu \mathrm{M})\end{array}$ & $\begin{array}{l}k_{\mathrm{cat}} / K_{\mathrm{M}} \\
\left(\mathrm{M}^{-1} \mathrm{~s}^{-1}\right) \\
\end{array}$ & $\begin{array}{c}\mathrm{MMH} / \mathrm{DMH} / \mathrm{TMH} \\
\text { ratio (\%) } \\
\end{array}$ \\
\hline HIS[b] & EgtD & $5.8 \times 10^{-1}$ & 110 & $5.3 \times 10^{3}$ & \\
\hline $\mathrm{DMH}[\mathrm{b}]$ & EgtD & $4.3 \times 10^{-1}$ & 32 & $1.7 \times 10^{4}$ & $<1 / 17 / 83$ \\
\hline Tyr[b] & Ybs & $1.1 \times 10^{-1}$ & 21 & $5.2 \times 10^{3}$ & \\
\hline DMY & Ybs & $5.0 \times 10^{-2}$ & 43 & $1.2 \times 10^{3}$ & $<1 / 60 / 40$ \\
\hline $\operatorname{Trp}[\mathrm{b}]$ & EgtDE282A,M252V & $1.1 \times 10^{-1}$ & 20 & $5.5 \times 10^{3}$ & \\
\hline DMW & EgtD $_{\text {E282A,M252V }}$ & $0.9 \times 10^{-2}$ & 11 & $8.2 \times 10^{2}$ & $<1 / 95 / 5$ \\
\hline
\end{tabular}

[a] Reaction conditions: $25^{\circ} \mathrm{C}$, Tris $\mathrm{HCl} 50 \mathrm{mM}, \mathrm{pH}$ 8, NaCl $50 \mathrm{mM}, \mathrm{MnCl} 200 \mu \mathrm{M}, 500 \mu \mathrm{M}$ SAM, SAH nucleosidase $5 \mu \mathrm{M}$, adenine deaminase $10 \mu \mathrm{M}$

[b] Data from Ref. 33

Structure of EgtD in complex with TMH. In order to mediate efficient trimethylation and to allow for product inhibition, EgtD must be able to bind to a primary, a secondary, a tertiary and a quaternary amine. How can this enzyme accommodate the changing hydrogen-bonding requirements of its ligand? To examine this question, we solved the crystal structure of EgtD in complex with TMH (Table S1 \& S2). This structure superimposes with the EgtD:HIS 50 and the EgtD:DMH ${ }^{33}$ complexes a mutual r.m.s.d. of 0.4 Å (entire chain). The three ligands HIS, DMH and TMH occupy essentially the same position and almost all atoms of the active site residues superimpose. The important exceptions are residues in direct contact with the $\alpha$-amino moiety. These residues are Asn166 and Gly161.

In the TMH complex two $\mathrm{N}-\alpha$-methyl groups make close contact with the backbone carbonyl of Gly161 (3.1 ̊) and with the side chain carbonyl of Asn166 (3.2 ̊). The carbonyls approach the N- $\alpha$ methyl groups in a (O-C-N $\alpha$ ) angle of $167^{\circ}$ (Gly161) and $177^{\circ}$ (Asn166) This geometry is suggestive of attractive interaction between the positively polarized methyl groups and the carbonyl functions. The third $\mathrm{N}$ - $\alpha$-methyl group points toward the SAM-binding site.

In the DMH complex, one $\mathrm{N}-\alpha$-methyl group makes the same interaction with the Gly161 carbonyl function ( $d=3.0 \AA$ ) and the other $N$ - $\alpha$-methyl group points toward the SAM-binding site. Asn166 moved $1.3 \AA$ closer to the substrate to form a $2.7 \AA$ hydrogen bond to the $\alpha$-amino group of the ligand. In the HIS complex the ligand forms two rather loose hydrogen bonds to Asn166 (3.1 Å) and Gly161 (3.4 ̊̊). In order to establish a hydrogen bond, the Gly161 carbonyl oxygen moved by $1.8 \AA$ towards the $\alpha$-amino group of HIS. This rearrangement is made possible by conformational changes of the backbone including residues 159 - 162. 
The three structures show that EgtD solvates the $\mathrm{N}$ - $\alpha$-amino moiety of its substrates and product by a highly polar pocket with adaptable size. Remarkably, HIS is by far the weakest binder, despite forming two classical hydrogen bonds (Table 2). ${ }^{33}$ In the DMH complex the hydrogen bond to Gly161 is lost, but the hydrogen bond to Asn166 becomes shorter and hence, stronger. As a consequence, $\mathrm{DMH}$ is a 100 -fold stronger EgtD ligand $\left(K_{\mathrm{D}}=4 \mu \mathrm{M}\right)$ than HIS. Surprisingly, TMH is still a comparatively strong ligand $\left(K_{\mathrm{i}}=40 \mu \mathrm{M}\right)$ even though both hydrogen bonds are lost. It is possible that the close interaction between two N- $\alpha$-methyl groups and the carbonyls from Gly161 and Asn166 are attractive and partially compensate for the lack of hydrogen bonding.

Figure 5. Crystal structures of EgtD in complex A) HIS (PDB entry 4UY7, 50); B) DMH (PDB entry 4PIN, ${ }^{33}$ ); C) TMH; D) 8; E) 2; F) 3 . Unbiased $m\left|F_{o b s}\right|-D\left|F_{c a l c}\right|$ electron density $(\sigma$-level $=2)$ of the compounds is shown in green.

Catalytic cycle. The structure of the EgtD:TMH complex also illustrates why TMH is necessarily a competitive inhibitor with respect to both HIS and SAM. A model of EgtD in complex with SAM and TMH indicates that the third $\mathrm{N}-\alpha$-methyl groups of TMH and the sulfonium methyl group of SAM would clash (Figure S7). Hence, binding to the two ligands is mutually exclusive. In the conformation of DMH observed in the EgtD:DMH complex the same steric clash would prevent binding of SAM. In order to form the ternary complex DMH (a, Figure 6) must first adopt an alternative conformation in which the two N- $\alpha$-methyl groups point towards Asn166 and Gly161 (b). This conformer can accept SAM (c) to form the reactive complex that decays via S- to N-methyl transfer to form the product complex (d). Based on this mechanistic model we hypothesized that DMH analogs that make the same interactions in the active site but cannot undergo the same conformational change could be potent EgtD inhibitors.

Figure 6. Mechanism of EgtD-catalyzed methylation of DMH. Residues Asn166 and Gly161 are shown in gray.

Strategies for inhibitor design. To test this idea we synthesized histidine derivatives with cyclic tertiary amines in place of the $\mathrm{N}$ - $\alpha$-dimethyl amino moiety of DMH (Figure 5, see supporting information). The syntheses and characterization of compounds depicted in Figure 7 are described in the supporting information. The inhibitory activities were measured using the same assay as described above. Consistent with the design strategy compounds 1, 2, and $\mathbf{3}$ were not methylated by EgtD, but instead inhibit EgtD-catalyzed methylation of HIS (Figure S8 \& S9, Table 1). In the presence of $0.5 \mathrm{mM} \mathrm{SAM}$, inhibition by $\mathbf{1}, \mathbf{2}$, and 3 is characterized by inhibition constants $\left(K_{\mathrm{i}}\right)$ of 9,40 and 90 $\mu \mathrm{M}$ respectively (Table 1). Compounds 1, $\mathbf{2}$ and $\mathbf{3}$ were synthesized in racemic form. Given that EgtD only interacts with L-amino acids, it is fair to assume that only the L-isomers of the inhibitors would bind (Figure S10). This assumption is corroborated by the finding that the pure L-form of $\mathbf{2}$ inhibits EgtD with a two-fold lower $K_{\mathrm{I}}$ than measured for the racemic mixture (Table 1). 
Figure 7. Structure of tested EgtD inhibitors.

Avoiding competition with SAM. As an alternative design strategy we examined inhibitors that only compete with HIS, but do not compete with SAM. Such compounds could form inhibited complexes with EgtD that are not destabilized by the generally high cellular concentration of SAM. To test this idea we synthesized racemic histidine derivatives with a proton (4), a methyl group (5), a hydroxyl group (6), fluoride (7), chloride (8) or bromide (9) in place of the $\alpha$-amine group. In kinetic assays these compounds, except for $\mathbf{5}$, proved stronger HIS-competitive inhibitors than TMH (Table 1). The inhibition constant of the L-form of $\mathbf{8}$ was again two-fold lower than that of the racemic form, indicating that the active site selectively binds one isomer. Determination of the inhibition mechanism of chlorohistidine (8, Figure 4) revealed HIS-competitive and SAM-uncompetitive inhibition, suggesting that the EgtD: 8 complex can still bind SAM.

The crystal structure of EgtD in complex with $\mathbf{8}$ confirms that this ligand occupies the same active site position as all other co-crystallized histidine derivatives (Figure 5). Unlike the $\alpha$-amine substituents in HIS, DMH, TMH, 2 and $\mathbf{3}$ the chloride substituent makes no direct contact with any protein residue. Solvation of the carboxylate and the imidazole ring by the active site apparently provides enough attractive interaction to induce strong inhibitory activity of the methyl- (5), chloro(8) and bromo-substituted derivatives (9). The lower affinities of compounds 4, 6 and $\mathbf{7}$ are most likely due to stronger solvation of the free ligand by water. The relatively poor affinity of EgtD for 6 mirrors the low affinity for HIS and corroborates the notion that the residues Gly161 and Asn166 are not particularly well positioned to engage in hydrogen bonding. 
Bisubstrate inhibitors. A more common strategy to design inhibitors for methyltransferases targets the SAM-binding pocket. One successful way to increase the specificity towards one particular type of methyltransferase is to integrate structural motifs from SAM with those of the specific methyl acceptor into a single bisubstrate inhibitor. ${ }^{51-56}$ To explore this approach for the design of EgtD inhibitors we synthesized four histidine derivatives $(\mathbf{1 0}-\mathbf{1 3})$ that are N-substituted to mimic the methionyl moiety of SAM. All four compounds displayed weak inhibitory activity (Table 1, Figure S11). One explanation could be that that the amino acid substituents are not recognized by the methionyl-binding site in EgtD. Indeed, reinspection of EgtD in complex with DMH and SAH showed the possibility that the chosen methionyl mimics may be too short to bridge the histidine binding site and the methionyl-binding site. It is also possible that the compounds do bind as intended, but that recognition of the methionyl-moiety does not produce enough attractive interaction to outweigh competition with SAM and HIS. The observation that the additional $\mathrm{N}-\alpha$-methyl group on compounds $\mathbf{1 1}$ and $\mathbf{1 3}$ increase the affinity by four to eight-fold provides further evidence that tertiary amines bind more strongly to EgtD than secondary amines.

Feedback inhibition. EgtD catalyzes the first step in EGT biosynthesis (Figure 1). This reaction converts the primary metabolites HIS and SAM to TMH as a substrate for the subsequent enzyme EgtB. Methyltransferases are very common contributors to biosynthetic pathways in natural product biosynthesis. ${ }^{57}$ However, a cursory inspection of all methyltransferase entries in the Braunschweig Enzyme Database (BRENDA) shows that SAM-dependent methyl transfers rarely occur as first biosynthetic steps..$^{58}$ For example, alkaloids or phenylpropanoids usually receive methyl groups at later biosynthetic stages. ${ }^{59-62}$

The unique role of EgtD as the gateway to EGT production raises the specific problem of regulation. Two studies on a gliotoxin deficient strain of A. fumigatus, and a mycothiol-deficient strain of $M$. smegmatis revealed that these deficiencies are compensated by EGT overproduction. ${ }^{63,64}$ The mechanism by which ETG productivity is coupled to seemingly unrelated biosynthetic activity is not known. However, the two studies provide first indications that EGT production may be regulated.

Because methyl transfer from SAM to His is essentially irreversible, and because both substrates are abundant metabolites, regulation of EgtD activity is essential, either by transcriptional control, by reversible inhibition or by destruction of the enzyme. One regulatory mechanism has been proposed based on the finding that EgtD from Mycobacterium tuberculosis may be a substrate of the protein kinase PknD. ${ }^{31}$ According to this model the kinase phosphorylates a key active site residue of EgtD (Thr213) to block activity. 
Proofreading. In addition to making the first intermediate in EGT biosynthesis, EgtD also serves as a quality control element of this pathway. Although EGT has been identified from a large range of sources, $1,4,5,23$ there are no isolation reports of EGT derivatives that lack one, two or all $\mathrm{N}$ - $\alpha$-methyl groups. For reasons that are not exactly clear the betaine moiety of EGT is important for physiological function. Subsequent enzymes in the EGT pathway are unable to proofread the methylation state of their substrates. ${ }^{2,36,37}$ For example, EgtB from M. smegmatis turns over DMH and TMH with almost the same efficiency. ${ }^{2}$ EgtC and EgtE are unlikely to prevent alternative products because the reaction catalyzed by EgtB is irreversible. Hence, the only mechanism to prevent the formation of unwanted EGT derivatives is to limit the cellular concentration of DMH. Limiting this concentration is an important role of EgtD. As shown in Table 2 EgtD binds HIS, MMH and DMH with increasing affinity. As a result, each added methyl group on the methyl acceptor increases the probability of further methylation. The three methyl groups are transferred in a cooperative process that avoids the accumulation of MMH or DMH. ${ }^{33}$ By contrast, the catalytic efficiency of the homologous enzymes Ybs and EgtD ${ }_{\mathrm{E} 282 \mathrm{~A}, \mathrm{M} 252 \mathrm{~V}}$ drops significantly after the first two methyl transfers to Tyr ad Trp, respectively (Table 3). Comparisons of the EgtD structure with the homology model of Ybs, and the crystal structure of EgtD $\mathrm{E}_{\mathrm{E} 282 \mathrm{~A}, \mathrm{M} 252 \mathrm{~V}}$ do not reveal clear structural explanations for these different activities. One possibility is that transfer of the last methyl group is particularly sensitive to precise positioning of the $\mathrm{N}$ - $\alpha$-dimethylated amino acid in the active site. Therefore, it is possible to 
conclude that the ability of EgtD to catalyze cooperative trimethylation is also an essential and specifically evolved feature.

Mechanistic implications. Finally, we would like to summarize what can be learned about the catalytic mechanism and about inhibitor design from the ligand binding preferences of EgtD. EgtD can form up to thirteen binary and ternary complexes with its six native ligands HIS, MMH, DMH, TMH, SAM and SAH (Figure 8). Among these, EgtD:DMH and EgtD:TMH:SAH are the strongest binary and ternary complexes (Table 2). The interaction between the amide side chain of Asn166 and the $\alpha$ amino function of DMH shows that the $\alpha$-amino function of the methyl acceptor is protonated in the binary complex (Figure 5). Apparently, the active site stabilizes a cationic moiety in this position. Unreactive histidine derivatives with neutral substituents (5, 8 and 9) form strong complexes with EgtD and SAM, suggesting that in the ternary complex the sulfonium moiety of SAM fully satisfies the requirement for a cationic charge in the active site. Consequently, the methyl acceptors HIS, MMH and DMH must lose a proton before or concomitant to SAM binding. Also, in order to make room for the second substrate, deprotonation must be accompanied by inversion of the $\alpha$-amine. For example, DMH must turn the two N- $\alpha$-methyl groups towards Gly161 and Asn166 in order to juxtapose the nucleophilic lone pair with the sulfonium methyl group of SAM (Figure 6). Each methyl transfer from HIS to TMH makes the ligand larger. The structures of EgtD in complex with HIS, DMH and TMH show how the active site undergoes stepwise expansion by repositioning of Gly161 and Asn166 to accommodate the growing size of the ligand.

To support efficient trimethylation the energy landscape of this expansion must be adjusted to increase the affinity for the methyl acceptor with each additional $\mathrm{N}-\alpha$-methyl group. As the thermodynamic binding data shows, EgtD follows exactly this expected behavior. A plot of the complex stabilities $\left(\Delta \mathrm{G}_{\text {binding }}\right)$ of EgtD with SAH and HIS, DMH or TMH shows that each additional methyl group on the methyl acceptor increases the complex stability by $1.2 \mathrm{kcal} / \mathrm{mol}$ (Figure 8, Table 2). A similar trend is apparent in the absence of SAH. Notably, the EgtD:TMH complex deviates from this trend. It seems possible that the stability of the EgtD:TMH complex is purposefully decreased to avoid inhibition by sub-micromolar concentrations of TMH.

A glance at the enthalpic and entropic contributions to the stability of the six complexes cautions that a purely structural interpretation of the binding data may be misleading (Table 2). For example, formation of the EgtD:DMH:SAH complex liberates more heat than formation of the EgtD:TMH:SAH complex. However, because the latter suffers almost no entropic penalty, the TMH complex is 20 -fold more stable. The enthalpic term indicates that formation of a hydrogen bond to Asn 166 and stacking one N- $\alpha$-methyl group towards Gly161 in the EgtD:DMH:SAH complex amounts to more attraction 
Figure 8. Top: Complete reaction scheme of EgtD catalyzed trimethylation of HIS. EgtD can combine with its six native ligands to 13 binary and ternary complexes. Bottom: the stability of EgtD complexes as determined by ITC (Table 2).

Conclusion. This report describes the unusual substrate binding mechanism of the SAM-dependent methyltransferase EgtD. Unlike most methyltransferases, this enzyme follows an obligatory sequential binding mechanism with the methyl acceptor as the leading substrate. Secondly, this enzyme can regulate EGT production by way of product inhibition. Third, the enzyme ensures efficient permethylation of its substrate and suppresses the accumulation of mono- and dimethylated intermediates. Product inhibition and efficient permethylation are the result of specific evolutionary optimization. These findings were exploited to design three types of substrate competitive EgtD inhibitors. The most efficient inhibitors (5 \& 8) are very simple histidine derivatives that provide promising leads for further development EGT biosynthesis inhibitors.

Acknowledgements. The authors would like to thank the BESSY II Synchrotron (Helmholtz Centre Berlin, Berlin, Germany) for providing beamline access. This project was supported by the Swiss National Science Foundation, the University of Basel, the "Professur für Molekulare Bionik" and a starting grant from the European Research Council (ERC-2013-StG 336559). A. Vit was supported by the HZI Graduate School for Infection Research.

Supporting Information Available: Detailed descriptions of all experiments, supporting figures (S1 - S11), schemes (S1 - S8) and tables (S1 - S2) are shown in the supporting information. This material is available free of charge via the internet at http://pubs.acs.org.

References

[1] Genghof, D. S., Inamine, E., Kovalenko, V., and Melville, D. B. (1956) Ergothioneine in microorganisms, J. Biol. Chem. 223, 9 17.

[2] Seebeck, F. P. (2010) In vitro reconstitution of Mycobacterial ergothioneine biosynthesis, J. Am. Chem. Soc. 132, 6632-6633.

[3] Pfeiffer, C., Surek, B., Schöming, E., and Gründemann, D. (2011) Cyanobacteria produce high levels of ergothioneine, Food Chem. 129, 1766 - 1769. 
[4] Alamgir, K. M., Masuda, S., Fujitani, Y., Fukuda, F., and Tani, A. Production of ergothioneine by Methylobacterium species, Front. Microbiol. 6, 1185.

[5] Burn, R., Misson, L. E., Meury, M., and Seebeck, F. P. (2017) Anaerobic Origin of Ergothioneine, Angew Chem Int Ed Engl. 56, $12508-12511$.

[6] Jones, G. W., Doyle, S., and Fitzpatrick, D. A. (2014) The evolutionary history of the genes involved in the biosynthesis of the antioxidant ergothioneine, Gene 549, 161-170.

[7] Gründemann, D., Harlfinger, S., Golz, S., Geerts, A., Lazar, A., Berkels, R., Jung, N., Rubbert, A., and Schoemig, E. (2005) Discovery of the ergothioneine transporter, Proc. Natl. Acad. Sci. U. S. A. 102, 5256-5261.

[8] Melville, D. B., and Eich, S. (1956) The occurrence of ergothioneine in plant material, J. Biol. Chem. 218, 647 - 651.

[9] Servillo, L., DOnofrio, N., and Balestrieri, M. L. (2017) Ergothioneine products derived by superoxide oxidation in endothelial cells exposed to high-glucose, J. Cardiovasc. Pharmacol. 69, 183 - 191.

[10] Melville, D. B. (1959) Ergothioneine, VITAMINS AND HORMONES-ADVANCES IN RESEARCH AND APPLICATIONS 17, 155 204.

[11] Halliwell, B., Cheah, I. K., and Drum, C. L. (2016) Ergothioneine, an adaptive antioxidant for the protection of injured tissues? A hypothesis, Biochem. Biophys. Commun. 470, 245 - 250.

[12] Kerley, R. N., McCarthy, C., Kell, D. B., and Kenny, L. C. (2017) The Potential Therapeutic Effects of Ergothioneine in Preeclampsia, Free Radic Biol Med. Epub ahead of print.

[13] Cumming, B. M., Lamprecht, D. A., Wells, R. M., Saini, V., Mazorodze, J. H., and Steyn, A. J. C. (2014) The Physiology and Genetics of Oxidative Stress in Mycobacteria, Microbiol. Spectr. 2.

[14] Cheah, I. K., and Halliwell, B. (2012) Ergothioneine; antioxidant potential, physiological function and role in disease, Biochim. Biophys. Acta. 1822, 784-793.

[15] Asmus, K. D., Bensasson, R. V., Bernier, J. L., Houssin, R., and Land, E. J. (1996) One-electron oxidation of ergothioneine and analogues investigated by pulse radiolysis: redox reaction involving ergothioneine and vitamin C, Biochem. J. 315, 625-629.

[16] De Luna, P., Bushnell, E. A., and Gauld, J. W. (2013) A Density Functional Theory Investigation into the Binding of the Antioxidants Ergothioneine and Ovothiol to Copper, J Phys Chem A 117, 4057 - 4065.

[17] Melnick, J. G., and Parkin, G. (2007) Cleaving Mercury-Alkyl Bonds: A Functional Model for Mercury Detoxification by MerB, Science 317, 225 - 227.

[18] Servillo, L., Castaldo, D., Casale, R., D'Onofrio, N., Giovane, A., Cautela, D., and Balestrieri, M. L. (2015) An uncommon redox behavior sheds light on the cellular antioxidant properties of ergothioneine, Free Radic. Biol. Med. 79, 228 - 236.

[19] Brummel, M. C. (1985) In search of a physiological function for L-ergothioneine, Med. Hypotheses 18, 351 - 370.

[20] Stoffels, C., Oumari, M., Perrou, A., Termath, A., Schlundt, W., Schmalz, H. G., Schäfer, M., Wewer, V., Metzger, S., Schömig, E., and Gründemann, D. (2017) Ergothioneine stands out from hercynine in the reaction with singlet oxygen: Resistance to glutathione and TRIS in the generation of specific products indicates high reactivity, Free Radic Biol Med. 113, 385 - 394.

[21] Arduini, A., Eddy, L., and Hochstein, P. (1990) The reduction of ferryl myoglobin by ergothioneine: a novel function for ergothioneine., Arch. Biochem. Biophys. 15, 41 - 43.

[22] Zhu, B.-Z., Mao, L., Fan, R.-M., Zhu, J.-G., Zhang, Y.-N., Wang, J., Kalyanaraman, B., and Frei, B. (2011) Ergothioneine Prevents Copper-Induced Oxidative Damage to DNA and Protein by Forming a Redox-Inactive ErgothioneineCopper Complex, Chem. Res. Toxicol. 24, 30-34.

[23] Ey, J., Schomig, E., and Taubert, D. (2007) Dietary Sources and Antioxidant Effects of Ergothioneine, J. Agric. Food Chem. $55,6466-6474$.

[24] Cumming, B. M., Chinta, K. C., Reddy, V. P., and Steyn, A. J. C. (2018) Role of Ergothioneine in Microbial Physiology and Pathogenesis., Antioxid. Redox Signal. 28, 431 - 444.

[25] Bello, M. H., Barrera-Perez, V., Morin, D., and Epstein, L. (2012) The Neurospora crassa mutant NcDEgt-1 identifies an ergothioneine biosynthetic gene and demonstrates that ergothioneine enhances conidial survival and protects against peroxide toxicity during conidial germination, Fungal Genet. Biiol 49, 160-172.

[26] Nakajima, S., Satoh, Y., Yanashima, K., Matsui, T., and Dairi, T. (2015) Ergothioneine protects Streptomyces coelicolor A3(2) from oxidative stresses, J. Biosci. Bioeng. 120, 294 - 298.

[27] Sheridan, K. J., Lechner, B. E., Keeffe, G. O., Keller, M. A., Werner, E. R., Lindner, H., Jones, G. W., Haas, H., and Doyle, S. (2016) Ergothioneine Biosynthesis and Functionality in the Opportunistic Fungal Pathogen, Aspergillus fumigatus, Sci Rep. 6, 35306.

[28] Singh, A. R., Strankman, A., Orkusyan, R., Purwantini, E., and Rawat, M. (2016) Lack of mycothiol and ergothioneine induces different protective mechanisms in Mycobacterium smegmatis, Biochem. Biophys. Rep. 8, 100 - 106.

[29] Sao Emani, C., Williams, M. J., Van Helden, P. D., Taylor, M. J. C., Wiid, I. J., and Baker, B. (2018) Gamma-glutamylcysteine protects ergothioneine-deficient Mycobacterium tuberculosis mutants against oxidative and nitrosative stress, Biochem. Biophys. Res. Commun. 495, 147 - 178.

[30] Saini, H. S., Cumming, B. M., Guidry, L., Lamprecht, D. A., Adamson, J. H., Reddy, V. P., Chinta, K. C., Mazorodze, J. H., Glasgow, J. N., Richard-Greenblatt, M., Gomez-Velasco, A., Bach, H., Av-Gay, Y., Eoh, H., Rhee, K., and Steyn, A. J. C. (2016) Ergothioneine Maintains Redox and Bioenergetic Homeostasis Essential for Drug Susceptibility and Virulence of Mycobacterium tuberculosis, Cell Rep. 14, 572 - 585.

[31] Richard-Greenblatt, M., Bach, H., Adamson, J. H., Pena-Diaz, S., Li, W., Steyn, A. J. C., and Av-Gay, Y. (2015) Regulation of Ergothioneine Biosynthesis and Its Effect on Mycobacterium tuberculosis Growth and Infectivity, J. Biol. Chem. 290, $23064-23076$.

[32] Ishikawa, Y., and Melville, D. B. (1970) The Enzymatic a-N-Methylation of Histidine, J. Biol. Chem. 245, 5967 - 5973.

[33] Vit, A., Misson, L. E., Blankenfeldt, W., and Seebeck, F. P. (2015) Ergothioneine Biosynthetic Methyltransferase EgtD Reveals the Structural Basis of Aromatic Amino Acid Betaine Biosynthesis, Chembiochem 16, 119 - 125.

[34] Goncharenko, K. V., Vit, A., Blankenfeldt, W., and Seebeck, F. P. (2015) Structure of the Sulfoxide Synthase EgtB from the Ergothioneine Biosynthetic Pathway, Angew. Chem. Int. Ed. Engl. 54, 2821 - 2824. 
[35] Faponle, A. S., Seebeck, F. P., and de Visser, S. P. (2017) Sulfoxide Synthase versus Cysteine Dioxygenase Reactivity in a Nonheme Iron Enzyme, J. Am. Chem. Soc. 139, 9259 - 9270.

[36] Vit, A., Mashabela, G. T., Blankenfeldt, W., and Seebeck, F. P. (2015) Structure of the Ergothioneine-Biosynthesis Amidohydrolase EgtC, ChemBioChem 16, 1490 - 1496.

[37] Song, H., Hu, W., Naowarojna, N., Her, A. S., Wang, S. G., Desai, R., Qin, L., Chen, X., and Liu, P. (2015) Mechanistic studies of a novel C-S lyase in ergothioneine biosynthesis: the involvement of a sulfenic acid intermediate, Sci. Rep. 5.

[38] Pluskal, T., Ueno, M., and Yanagida, M. (2014) Genetic and Metabolomic Dissection of the Ergothioneine and Selenoneine Biosynthetic Pathway in the Fission Yeast, S. pombe, and Construction of an Overproduction System, PLoS One 9, e97774.

[39] Hu, W., Song, H., Sae Her, A., Bak, D. W., Naowarojna, N., Elliot, S. J., Qin, L., Chen, X., and Liu, P. (2014) Bioinformatic and Biochemical Characterizations of C-S Bond Formation and Cleavage Enzymes in the Fungus Neurospora crassa Ergothioneine Biosynthetic Pathway, Org. Lett. 16, 5382 - 5385.

[40] Askari, A., and Melville, D. B. (1962) The reaction sequence in ergothioneine biosynthesis: hercynine as an intermediate, J. Biol. Chem. 237, 1615-\&.

[41] Liao, C., and Seebeck, F. P. (2017) Convergent Evolution of Ergothioneine Biosynthesis in Cyanobacteria., Chembiochem $18,2115-2118$.

[42] Cook, P. F., and Cleland, W. W. (2007) Enzyme Kinetics and Mechanism, Garland Science Publishing, New York.

[43] Dorgan, K. M., Wooderchak, W. L., Wynn, D. P., Karschner, E. L., Alfaro, J. F., Cui, Y. Q., Zhou, Z. S., and Hevel, J. M. (2006) An enzyme-coupled continuous spectrophotometric assay for S-adenosylmethionine-dependent methyltransferases, Anal. Biochem. 350, 249-255.

[44] Martin, J. L., and McMillan, F. M. (2002) SAM (dependent) I AM: the S-adenosylmethionine-dependent methyltransferase fold, Curr. Opin. Stuct. Biol. 12, 783 - 793.

[45] Schubert, H. L., Blumenthal, R. M., and Cheng, X. (2003) Many paths to methyltransfer: a chronicle of convergence, Trends Biochem. Sci. 28, 329 - 335.

[46] Lorenz, N., Wilson, E. V., Machado, C., Schardl, C. L., and Tudzynski, P. (2007) Comparison of Ergot Alkaloid Biosynthesis Gene Clusters in Claviceps Species Indicates Loss of Late Pathway Steps in Evolution of C. fusiformis, Appl. Environ. Microbiol., 7185-7191.

[47] Liscombe, D. K., Louie, G. V., and Noel, J. P. (2012) Architectures, mechanisms and molecular evolution of natural product methyltransferases, Nat. Prod. Rep. 29, 1238 - 1250.

[48] Dirk, L. M. A., Flynn, E. M., Dietzel, K., Couture, J.-F., Trievel, R. C., and Houtz, R. L. (2007) Kinetic Manifestation of Processivity during Multiple Methylations Catalyzed by SET Domain Protein Methyltransferases, Biochemistry 46, 3905 - 3915.

[49] Kloor, D., and Osswald, H. (2004) S-Adenosylhomocysteine hydrolase as a target for intracellular adenosine action, Trends Pharmacol Sci. 25, 294 - 297.

[50] Jeong, J. H., Cha, H. J., Ha, S. C., Rojviriya, C., and Kim, Y. G. (2014) Structural insights into the histidine trimethylation activity of EgtD from Mycobacterium smegmatis, Biochem. Biophys. Res. Commun. 452, 1098 - 1103.

[51] Lerner, C., Ruf, A., Gramlich, V., Masjost, B., Zürcher, G., Jakob-Roetne, R., Borroni, E., and Diederich, F. (2001) X-ray Crystal Structure of a Bisubstrate Inhibitor Bound to the Enzyme Catechol-y- methyltransferase: A Dramatic Effect of Inhibitor Preorganization on Binding Affinity, Angew Chem Int Ed Engl. 40, 4040 - 4042.

[52] Ma, Z., Liu, H. W., and Wu, B. T. (2014) Structure-based drug design of catechol-0- methyltransferase inhibitors for CNS disorders, Br. J. Clin. Pharmacol. 77, 410 - 420.

[53] Zhang, G., Richardson, S. L., and Huang, R. (2015) Design, synthesis, and kinetic analysis of potent protein N-terminal methyltransferase 1 inhibitors, Org. Biomol. Chem. 13, 4149 - 4154.

[54] Hobley, G., McKelvie, J. C., Harmer, J. E., Howe, J., Oyston, P. C., and Roach, P. L. (2012) Development of rationally designed DNA N6 adenine methyltransferase inhibitors, Bioorg. Med. Chem. Lett. 22, $3079-3082$.

[55] Vaubourgeix, J., Bardou, F., Boissier, F., Julien, S., Constant, P., Ploux, O., Daffé, M., Quémard, A., and Mourey, L. (2009) SAdenosyl-N-decyl-aminoethyl, a Potent Bisubstrate Inhibitor

of Mycobacterium tuberculosis Mycolic Acid Methyltransferases, J. Biol. Chem. 284, 19321 - 19330.

[56] Osborne, T., Roska, R. L., Rajski, S. R., and Thompson, P. R. (2008) In Situ Generation of a Bisubstrate Analogue for Protein Arginine Methyltransferase 1, J. Am. Chem. Soc. 130, 4574 - 4575.

[57] Struck, A. W., Thompson, M. L., Wong, S. L., and Micklefield, J. (2012) S-Adenosyl-Methionine-Dependent Methyltransferases: Highly Versatile Enzymes in Biocatalysis, Biosynthesis and Other Biotechnological Applications, Chembiochem 13, 2642 - 2655.

[58] Placzek, S., Schomburg, I., Chang, A., Jeske, L., Ulbrich, M., Tillack, J., and D., S. (2017) BRENDA in 2017: new perspectives and new tools in BRENDA, Nucleic Acids Res. 45, D380 - 388.

[59] Vogt, T. (2010) Phenylpropanoid biosynthesis., Molecular Plant 3, 2 - 20.

[60] Jakubczyk, D., Cheng, J. Z., and O'Connor, S. E. (2014) Biosynthesis of the ergot alkaloids., Nat. Prod. Rep. 31, 1328 - 1338.

[61] Xu, W., Gavia, D. J., and Tang, Y. (2014) Biosynthesis of fungal indole alkaloids., Nat. Prod. Rep. 31, 1474 - 1487.

[62] Ziegler, J., and Facchini, P. J. (2008) Alkaloid biosynthesis: metabolism and trafficking., Annu. Rev. Plant Biol 59, 735 - 769.

[63] Gallagher, L., Owens, R. A., Dolan, S. K., O'Keeffe, G., Schrettl, M., Kavanagh, K., Jones, G. W., and Doyle, S. (2012) The Aspergillus fumigatus protein GliK protects against oxidative stress and is essential for gliotoxin biosynthesis., Eukaryot Cell. 11, 1226 - 1238.

[64] Ta, P., Buchmeier, N., Newton, G. L., Rawat, M., and Fahey, R. C. (2011) J. Bacteriol. 193, 1981-1990.

[65] Orr, E. L., and Quay, W. B. (1978) Product inhibition of rat brain histamine-N-methyltransferase, J. Neurochem. 30, 1539 1542.

[66] Wu, Q., and McLeish, M. J. (2013) Kinetic and pH studies on human phenylethanolamine N-methyltransferase., Arch Biochem Biophys. 539, 1 - 8.

[67] Baron, R. A., and Casey, P. J. (2004) Analysis of the kinetic mechanism of recombinant human isoprenylcysteine carboxylmethyltransferase (Icmt), BMC Biochem. 5, 19. 
[68] De Luca, V., and Ibrahim, R. K. (1985) Enzymatic synthesis of polymethylated flavonols in Chrysosplenium americanum. II. Substrate interaction and product inhibition studies of flavonol 3-, 6-, and 4'-0-methyltransferases., Arch. Biochem. Biophys. 238, 596 - 605.

[69] Cleland, W. W. (1963) The kinetics of enzyme-catalyzed reactions with two or more substrates or products. III. Prediction of initial velocity and inhibition patterns by inspection., Biochim. Biophys. Acta 67, 173 - 187.

[70] Pakusch, A. E., and Matern, U. (1991) KineticCharacterizationofCaffeoyl-CoenzymeA-Specific 3-0-Methyltransferase from Elicited Parsley Cell Suspensions1, Plant. Physiol. 96, 327 - 330. 


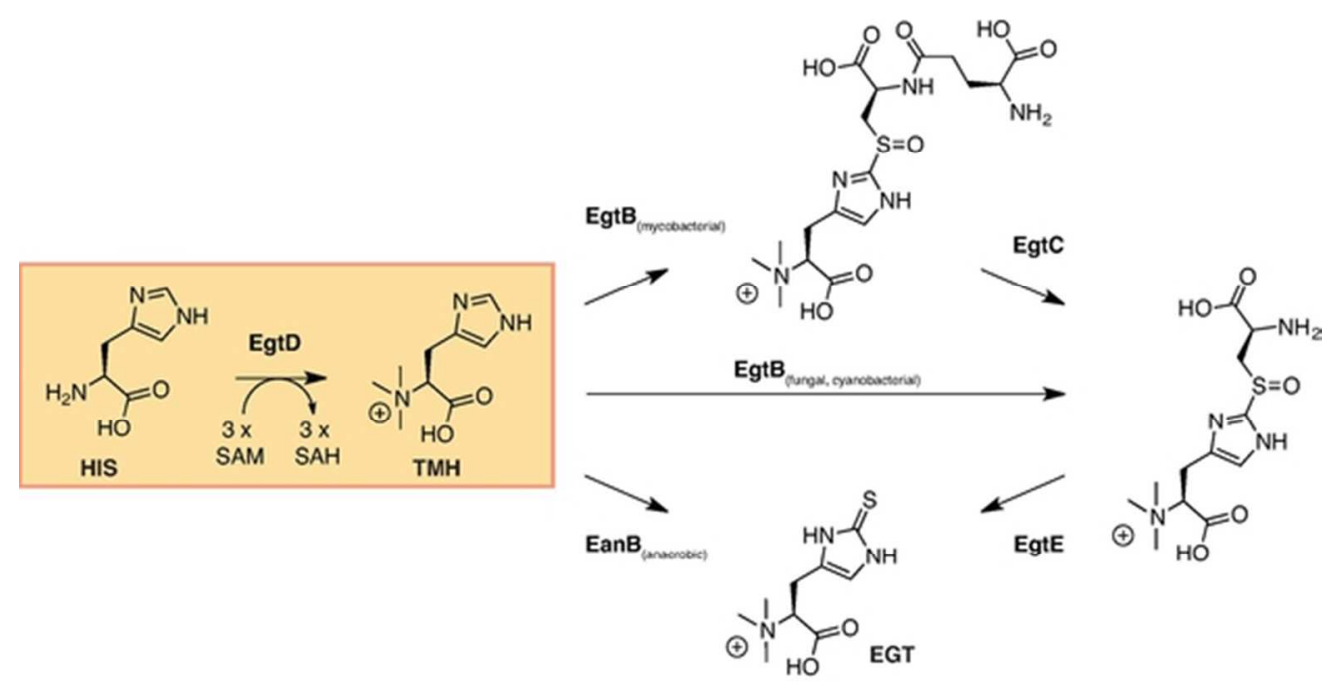

Four biosynthetic pathways for ergothioneine (EGT) production in Mycobacteria, fungi, cyanobacteria, and anaerobic green sulfur bacteria.

$52 \times 27 \mathrm{~mm}(300 \times 300 \mathrm{DPI})$ 

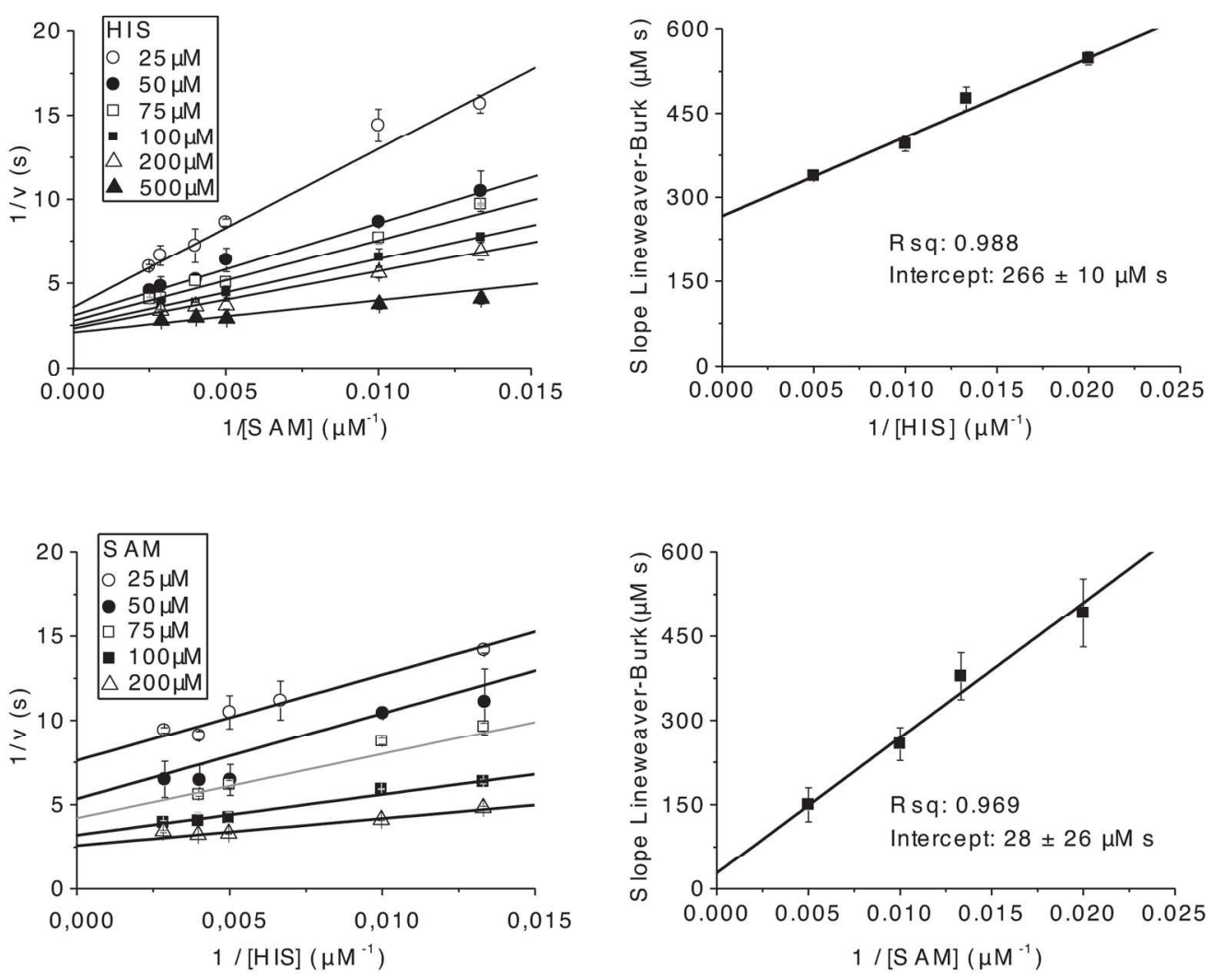

Lineweaver-Burk plots of the data used to examine the substrate binding mechanism of EgtD. Top: Primary and secondary plots with SAM as the variable substrate in presence of different concentrations of HIS. Bottom: Primary and secondary plots with HIS as the variable substrate in presence of different concentrations of SAM.

$165 \times 132 \mathrm{~mm}(300 \times 300 \mathrm{DPI})$ 

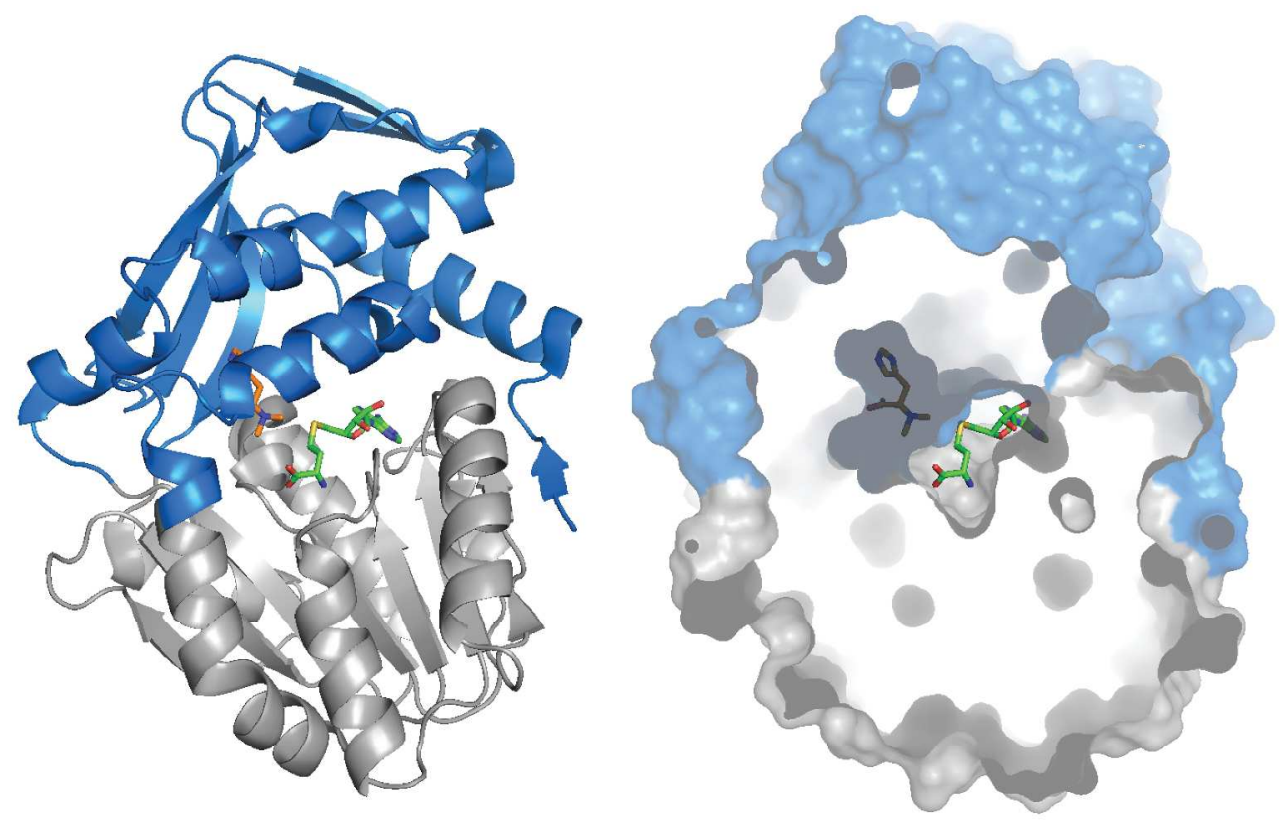

Structure of EgtD in complex with S-adenosyl homocysteine (SAH, green) and Na-dimethylhistidine (DMH, orange)(PDB: 4PIO). 5 The substrate-binding domain (blue) is formed by residues $1-60$ and $196-286$. The SAM-binding domain is conserved in most SAM-dependent methyltransferases.

$332 \times 219 \mathrm{~mm}(300 \times 300 \mathrm{DPI})$ 

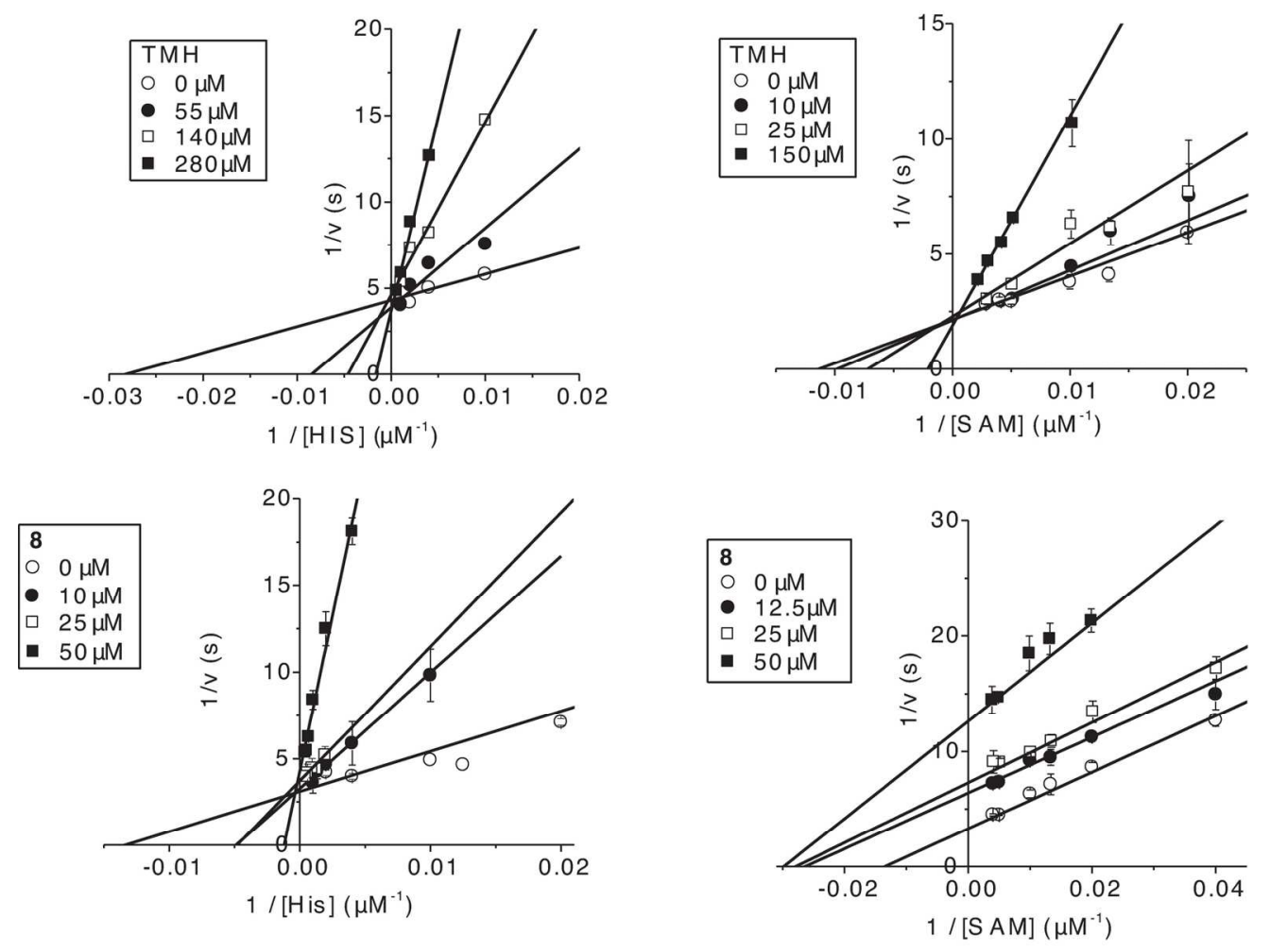

Lineweaver-Burk plots of the data used to examine EgtD inhibition by TMH and 8. Top: Primary plots with HIS or SAM as the variable substrate in presence of different concentrations of TMH Bottom: Primary plots with HIS or SAM as the variable substrate in presence of different concentrations of 8.

\section{$156 \times 119 \mathrm{~mm}(300 \times 300 \mathrm{DPI})$}



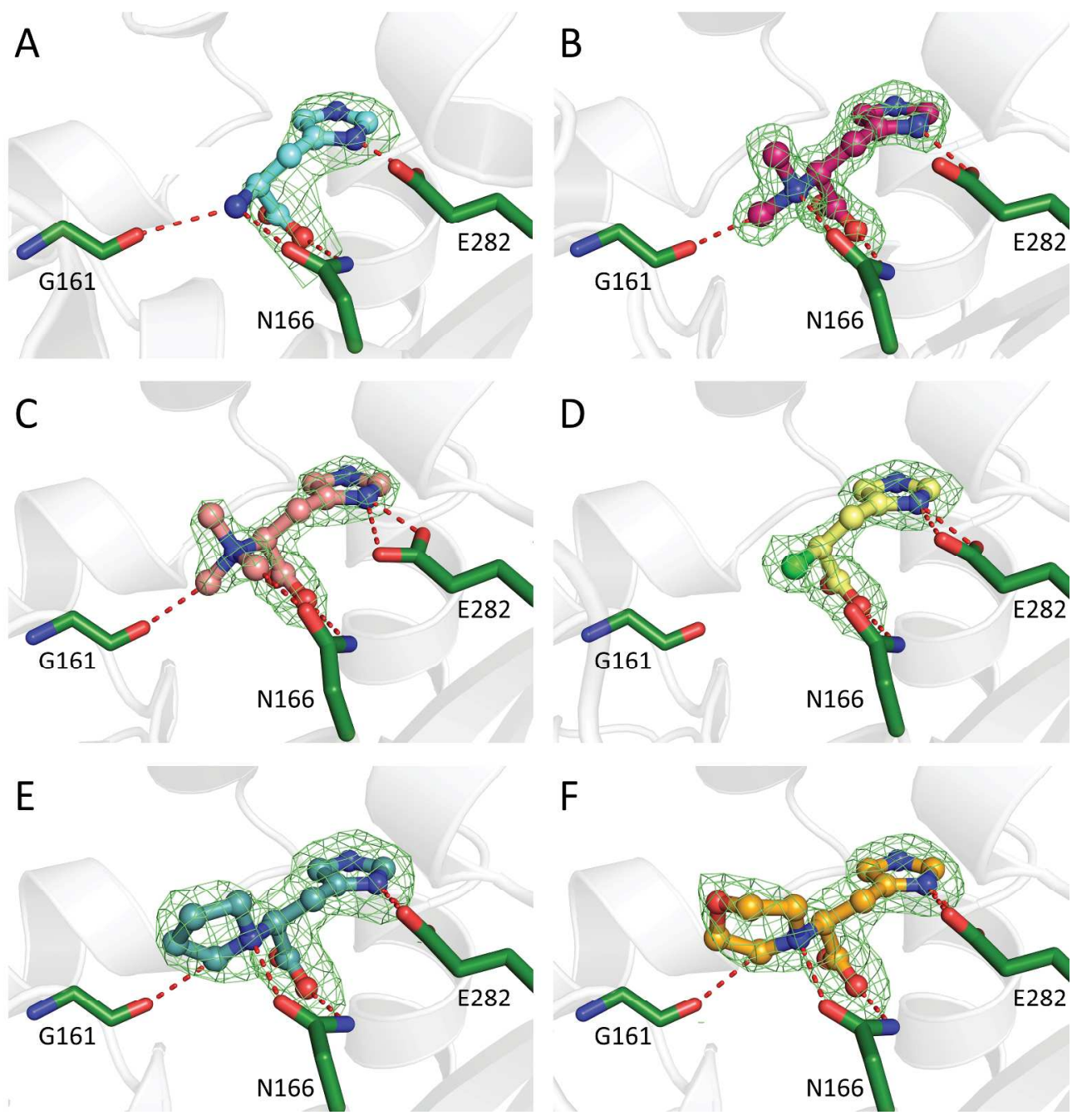

Crystal structures of EgtD in complex A) HIS (PDB entry 4UY7, 50); B) DMH (PDB entry 4PIN); C) TMH; D) 8 ; E) 2; F) 3 . Unbiased $m \mid$ Fobs $\mid$-D |Fcalc| electron density $(\sigma$-level $=2$ ) of the compounds is shown in green.

$514 \times 527 \mathrm{~mm}(600 \times 600$ DPI $)$ 
Mechanism of EgtD-catalyzed methylation of DMH. Residues Asn166 and Gly161 are shown in gray $31 \times 7 \mathrm{~mm}(300 \times 300 \mathrm{DPI})$ 


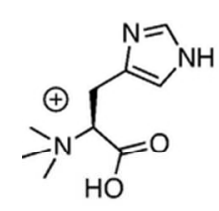

TMH

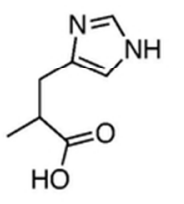

5

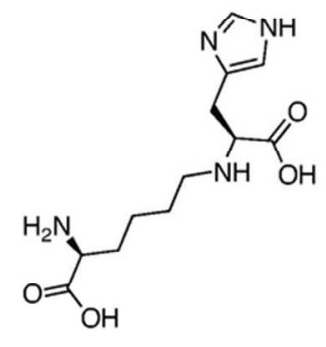

10<smiles>O=C(O)C(Cc1c[nH]cn1)N1CCC1</smiles>

1<smiles>O=C(O)C(O)Cc1c[nH]cn1</smiles>

6<smiles>O=C(O)C(Cc1c[nH]cn1)N1CCCC1</smiles>

2<smiles>O=C(O)C(F)Cc1c[nH]cn1</smiles>

7<smiles>O=C(O)C(Cc1c[nH]cn1)N1CCOCC1</smiles>

3<smiles>O=C(O)C(Cl)Cc1c[nH]cn1</smiles>

8<smiles>O=C(O)CCc1c[nH]cn1</smiles>

4<smiles>O=C(O)C(Br)Cc1c[nH]cn1</smiles>

9

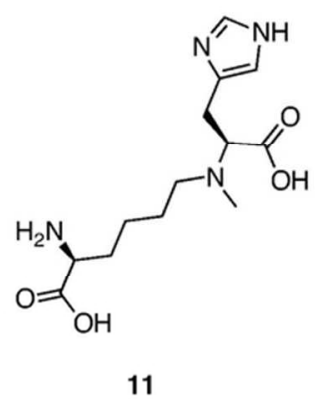

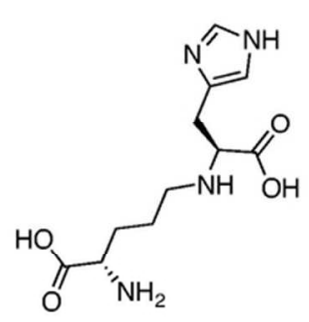

12

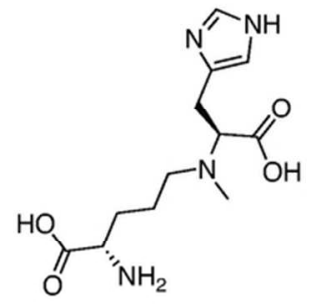

13

Structure of tested EgtD inhibitors.

$71 \times 57 \mathrm{~mm}(300 \times 300$ DPI $)$ 

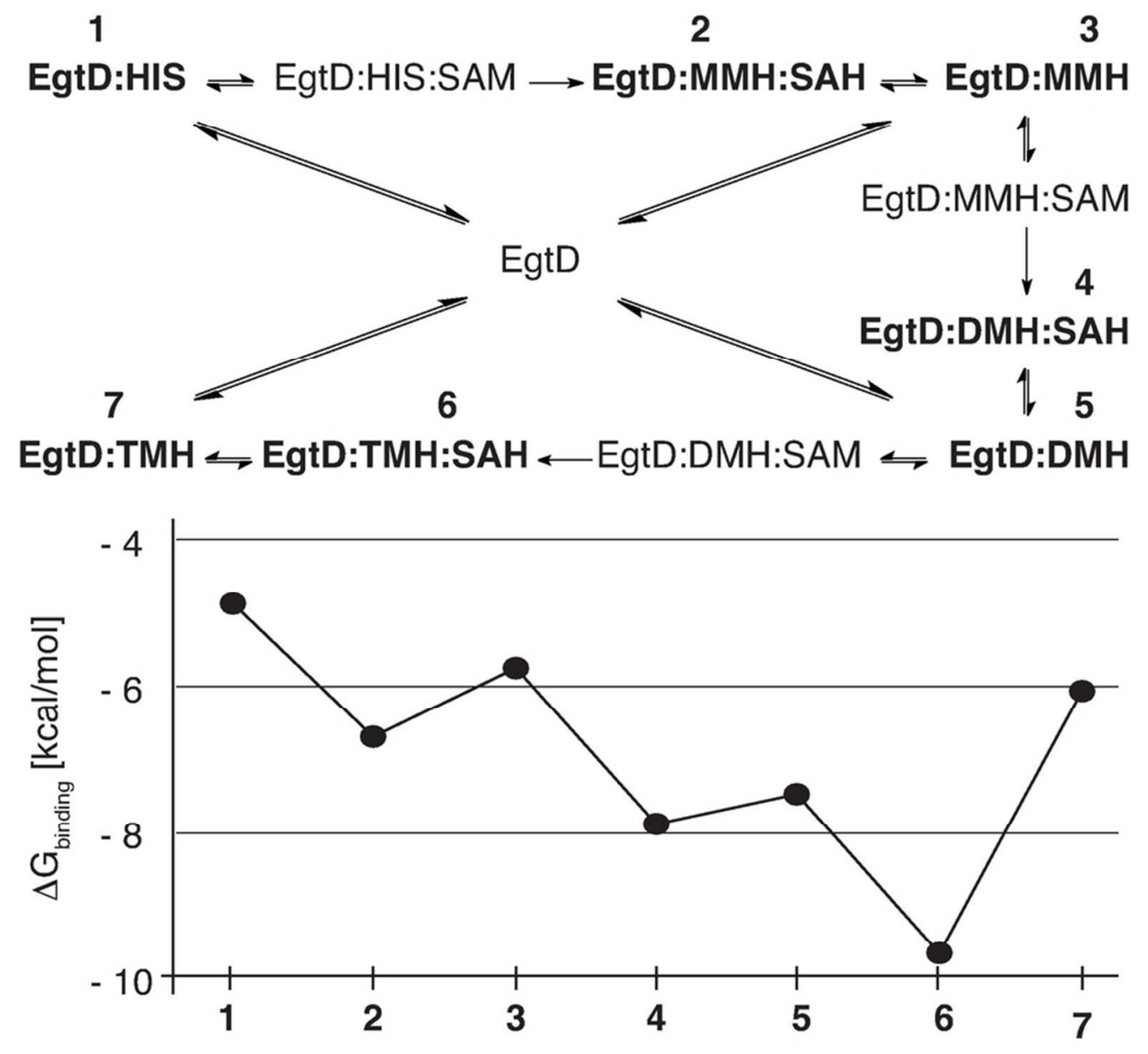

Top: Complete reaction scheme of EgtD catalyzed trimethylation of HIS. EgtD can combine with its six native ligands to 13 binary and ternary complexes. Bottom: the stability of EgtD complexes as determined by ITC (Table 2 ).

$80 \times 75 \mathrm{~mm}(300 \times 300$ DPI $)$ 


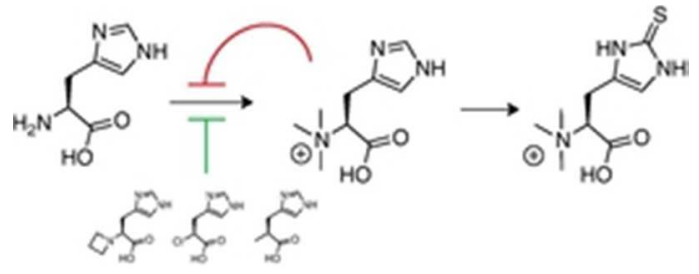

